\title{
Les clitiques accusatifs versus datifs dans les constructions causatives en faire
}

\author{
Béatrice Lamiroy \\ FRANITALCO, Université de Leuven \\ beatrice.lamiroy@arts.kuleuven.be \\ Michel Charolles \\ LATTICE, ENS Paris, Université de Paris-3 \\ michel.charolles@ens.fr
}

\section{Introduction}

Notre article a pour objet la construction causative en faire ${ }^{1}$, et en particulier la forme que prend le clitique qui renvoie au « contrôleur » de l'infinitif, celui-ci pouvant se mettre à l'accusatif (1) ou au datif (2) $:^{2}$
(1a) Paul a fait rire les étudiants
(1b) Paul les a fait rire
(2a) Je fais apprendre ses leçons à Paul
(2b) Je lui fais apprendre ses leçons

Du point de vue sémantique, les énoncés causatifs mettent toujours ${ }^{3}$ en jeu deux éléments au moins, une entité causatrice et une entité qui subit l'effet résultant de la causation. La situation causative est soumise à deux conditions essentielles : (a) l'évènement causé est temporellement postérieur à l'évènement causateur et (b) l'évènement causé est entièrement dépendant de l'occurrence de l'évènement causateur (Shibatani 1976 et 2002). Cela dit, plusieurs types de causation peuvent être distinguées en fonction du critère de « directness » (Dixon 2000 : 67), c'est-à-dire selon que l'effet causé est obtenu directement ou indirectement. Dans les exemples suivants, on observe en effet que dans (3a) il y a un lien direct entre la cause (le professeur) et l'effet (réfléchir), tandis que dans (3b), la cause (New York) réalise de manière indirecte (par son architecture) l'effet obtenu (évoquer Hong Kong) :

(3a) Le professeur fournit aux élèves des outils d'analyse pour l'image animée ; il les fait réfléchir à la problématique de l'adaptation d'une oeuvre littéraire ...

(3b) Aujourd'hui les enfants nous disent que New York les fait penser à Hong Kong !

Mardi : Statue de la Liberté, Ellis Island

Dans (3a), tant le sujet de faire que celui de l'infinitif sont agentifs, alors qu'ils ne le sont pas dans (3b). Entre ces deux extrêmes il existe sans doute un continuum causal (Shibatani 2002) allant du prototypiquement causatif à un effet affaibli de causation. Une des questions que nous poserons est de savoir s'il y a un lien entre le degré d'agentivité des sujets et la distribution des clitiques.

Du point de vue syntaxique, l'analyse classique (e.a. Blanche-Benveniste et al. 1987, Blanche-Benveniste 2007, Dannell 1979, Dixon 2000, Perlmutter 1983, Tasmowski 1985) consiste à considérer la structure causative comme un complexe verbal, ${ }^{4}$ c'est-à-dire un ensemble qui, contrairement à la structure infinitive à deux membres dont chacun peut avoir ses arguments (4), traite tous les arguments comme appartenant au verbe principal (5) :

(4a) Léa a prié Paul de répondre à la lettre

(4b) Léa l'a prié d'y répondre

(4c) *Léa l'y a prié de répondre

(5a) Léa a fait répondre Paul à la lettre 
Pour ce qui est des clitiques en français, la norme, signalée par les grammaires (Fournier, 1998 : 112 ; Grevisse-Goosse, 2008: §903, Riegel, 1996: 230) comme par les spécialistes (Blanche-Benveniste, 2007, Dannell, 1979 ; Kayne, $1977: 196$ ff ; Herslund, 1988 : 63), veut que le complément objet de faire se mette à l'accusatif quand le verbe qui suit faire est intransitif, au datif quand celui-ci est transitif, comme en (1-2) ci-dessus. ${ }^{6}$

Toutefois, comme le mentionnent la plupart des auteurs (Grevisse-Goosse, ibidem; Herslund, 1988 : 246, ; Dannell, 1979 ; Le Goffic, 1993 : 323 ; Kayne, $1977: 203)$, il existe des contre-exemples à la règle : on trouve le clitique datif avec des infinitifs intransitifs (6), et d'après Grevisse-Goosse surtout autrefois, l'accusatif avec des infinitifs transitifs (7):

(6a) Si vous croyez que c'est commode de lui faire changer d'idée (P.Benoît cité BU)

(6b) ... un coup (...) qui l'a pourtant suffisamment effrayé pour lui faire renoncer à son projet (A. Robbe-Grillet cité BU)

(6c) Elle lui fera téléphoner à ses parents (Kayne 1977 :203, note 9)

(6d) Il est vrai que la forme de ce gros nuage lui fait penser à une silhouette de cheval ailé

(7a) On ne la fera point dire ce qu'elle ne dit pas (Madame de Sévigné cité BU)

(7b) Il m'est impossible de le faire aborder ce sujet (A. Duhamel cité BU)

(7c) Les vrais Juifs et les vrais chrétiens ont toujours attendu un Messie qui les ferait aimer Dieu (Pascal).

(7d) Il se sent fatigué, comme s'il venait de s'éveiller d'une nuit agitée et trop courte, et le frottement de ses pantoufles sur le plancher de bois nu le fait penser à du papier d'émeri (Paul Auster, Dans le scriptorium)

Notons qu'on trouve parfois les deux clitiques dans des contextes identiques:

(8a) L'étonnement lui avait fait se dire tout bas ... (P. Bourget, cité Skårup 1985)

(8b) Cette admiration qui la faisait se dire ... (P. Bourget, ibidem)

(8c) Leur incarcération volontaire (...) les poussait à la mélancolie et leur faisait broyer du noir (Google)

(8d) Pour moi, les méthodes Ricci étaaient trop violentes, elle déstabilisait les élèves,

les faisait broyer du noir (Google)

Quand on consulte un moteur de recherche comme Google, on observe que les deux clitiques sont en effet attestés dans des cas contraires à la norme, ce qui suggère que la confusion semble concerner non seulement des stades plus anciens du français, mais la langue actuelle. ${ }^{7}$ Il est évident que l'utilisation de Google comme corpus demande des précautions, dans la mesure où on ne connaît pas l'identité des utilisateurs, qui peuvent en outre laisser des fautes de frappe. Une recherche sur Frantext sur une période récente et sur un corpus oral soigneusement transcrit serait évidemment nécessaire afin de vérifier l'hypothèse et fait partie de nos objectifs pour le futur. Ce que nous avons voulu faire ici est simplement attirer l'attention sur des données qui à première vue paraissent parfois déviants, mais qui pourraient être révélateurs, dans la mesure où on peut estimer qu'un locuteur qui utilise une structure aussi complexe que la causative en faire est quelqu'un qui manie le français avec une certaine compétence de la langue. Autrement dit, nous pensons que la confusion entre LE et LUI qu'on observe dans les données que nous allons discuter ci-dessous n'est pas a priori le fruit du hasard.

Même si les exemples suivants, où l'accusatif apparait avec des verbes prototypiquement transitifs tels manger, lire, construire, acheter, écrire ou sentir ne sont pas légion, ils sont bel et bien attestés sur Internet ${ }^{8}$

(9a) Mon fils de 7 mois et demi refusait le biberon du matin et du soir (...) et pour le soir plus de biberons je le fais manger un petit pot à base de laits et 2 petit suisses et tout se passe bien.

(9b) J'ai justement le livre "la cabane magique" : Panique à Pompéi, Le terrible empereur de Chine... Je le fais lire un chapitre quand il veut me faire plaisir. 
(9c) Une fois que j'ai suffisement d'or, je le fais construire un ziggourat. normalement, le tout ne prends pas plus de $2 \mathrm{~min}$.

(9d) Donc je le fais acheter un câble ADSL blindé de $15 \mathrm{~m}$ de long. Et on réessaye ; ça marche, mais coupé toutes les $5 \mathrm{mn}$ environ

(9e) Rédacteur en chef du journal « Le Matin », il la fait écrire quelques chroniques.

(9f) quand je veux couper les griffes, je couche mon chat, je le fais sentir la pince. ${ }^{9}$

De même, des exemples comme ceux qui suivent qui présentent le cas inverse, soit des datifs avec des verbes prototypiquement intransitifs tels déprimer, rigoler ou mourir, sont moins nombreux que ceux où le même verbe est précédé de faire + accusatif, mais ils sont attestés :

(10a) Puis, discrètement elle me dit que ça lui fait rigoler car elle adore qu'on soit brutal avec elle.

(10b) Son mec est venu l'embrasser et lui gratifier d'un cunni qui lui fait mourir d'excitation.

(10c) Si c'est vrai, c'est sûrement parce que ça leur fait déprimer d'en avoir une petite donc ils se rabattent sur la bouffe mais je pense que c'est une connerie

Notre objectif dans cet article est d'examiner ce genre de phrases de plus près afin de vérifier si on peut avancer une hypothèse qui rende compte de la distribution observée. Nous nous poserons en particulier la question de savoir si le choix du datif et de l'accusatif peut être motivé par des raisons sémantiques comme le degré d'agentivité du contrôleur de l'infinitif.

Notons d'emblée que nous excluons de notre recherche un certain nombre de structures apparentées:

- celle où l'objet de faire correspond à un SN lexical :

(11a) On a fait planter des choux aux linguistes (Herslund $1988: 238$ )

(11b) Je ferai partir John immédiatement (Kayne $1977: 196)$

(11c) En lui donnant un coup de pied, elle a fait faire un tour à la boite de chocolats ${ }^{10}$

- celle où le datif correspond non pas à l'agent mais au destinataire de l'infinitif (Herslund 1988 : 243, Hulk 1984, Tasmowski 1983, Tayalati 2008) ; cette construction présente des contraintes particulières illustrées en (12b) qui ne concernent pas le tour en cause ici :

(12a) On lui a fait parvenir le message

(12b) * On lui a fait téléphoner Marie

- celle où les clitiques correspondent à la 1 re ou 2e personne ou au pronom réfléchi puisque l'opposition entre datif et accusatif y est neutralisée :

(13a) C'est alors que Jean Paulhan m'a fait avoir un prix prestigieux (R-J. Clot, cité

Skårup 1985)

(13b) Fais-moi voir

(13c) Ils se sont fait sursauter mutuellement

- celle où l'agent est exprimé par un complément prépositionnel en par :

(14) On a fait planter les choux par les linguistes (Herslund $1988: 64$ )

- celle, plus complexe, où un pronom clitique s'associe au verbe faire, alors que d'autres clitiques sont attachés au verbe infinitif, par ex. Paul les a fait se laver, se rencontrer, se les présenter, s'en communiquer les modalités, etc. Ce genre de structures demanderait à être étudié de plus près, d'autant plus que le clitique accusatif semble s'imposer quand l'infinitif est pronominal, alors qu'on se trouve ici devant des verbes transitifs (ex. laver qq vs. se laver).

Nous souscrivons à l'analyse de Herslund $(1988: 240)^{11}$ pour qui le datif fonctionne comme thème et sujet secondaire de la prédication ['on a fait: les linguistes ont planté des choux'], alors que la construction causative en par $S N$ est analysée comme une structure ergative puisque c'est l'accusatif qui est thème et sujet de la prédication secondaire passive ['on a fait: les choux ont été plantés par les linguistes']. 
Notre article est organisé comme suit. Avant de passer aux données, nous présentons brièvement deux hypothèses avancées dans la littérature pour rendre compte de l'alternance des deux clitiques. Nous les vérifions ensuite en étudiant de plus près un petit échantillon d'exemples qu'on peut considérer comme une étude de cas et qui se limitent aux infinitifs penser et réfléchir.

\section{Hypothèses}

Dans la littérature, on trouve, en gros, deux hypothèses pour rendre compte de la confusion qui s'observe quant à la distribution des deux clitiques. L'une est d'ordre syntaxique (2.1.), l'autre propose une explication sémantique (2.2.).

2.1. Selon Dannell (1979 : 79), le datif apparaitrait avec un infinitif transitif indirect, là où on s'attendrait à un accusatif, quand le verbe admet dans sa valence à la fois un objet direct et un objet indirect. C'est le cas de verbes tels que croire, changer ou penser, par exemple. L'emploi du datif s'expliquerait alors par une contamination des deux constructions, la raison de la confusion serait donc avant tout syntaxique :

(15a) croire, penser à quelque chose vs croire, penser quelque chose
(15b) changer de quelque chose vs changer quelque chose
(16a) Mais toute tolérance accordée aux fanatiques leur fait croire immédiatement à
de la sympathie pour leur cause (M. Yourcenar, cité Skårup 1985)
(16b) Un élu UMP déclare que Ségolène Royal lui fait penser à sa femme de ménage
(Google)
(16c) Dans l'espoir de lui faire changer d'avis (Beja, cité Herslund 1988:246)

D'après le même auteur, la construction avec datif se serait progressivement étendue aux emplois avec un infinitif purement intransitif, comme dans les exemples ci-dessous (voir aussi ex. 10), de sorte qu'on aurait dans tous ces cas "un accusatif au niveau conceptuel mais un objet indirect au niveau syntaxique" :

(17a) Maman elle dit qu'ils vendent de la drogue (...) que c'est pour ça qu'ils tapent dans la main parce que la drogue, ça leur fait rigoler (Google)

(17b) Ils aiment bien se foutre la gueule d'eux-mêmes, ça leur fait rigoler (Google)

2.2. D'après Herslund (1990), il y aurait une généralisation du datif au détriment de l'accusatif pour des raisons sémantiques plutôt : avec le datif on assiste au «marquage de l'agent de V2 [= l'infinitif] pour bien souligner ce rôle d'agent et de sujet secondaire ». Cette hypothèse est séduisante dans la mesure où le datif est typologiquement reconnu (Lamiroy, 2000 ; Lazard, 1994; Van Belle et Van Langendonck, 1996-97) comme le cas le plus agentif, après le nominatif: c'est le «deuxième à bord» comme dit Herslund (1997).

Notons d'emblée que cette hypothèse est contredite cependant dans tous les cas où on observe l'accusatif avec des infinitifs transitifs directs, c'est-à-dire des cas où l'objet de faire a nécessairement le «rôle d'agent et de sujet secondaire » de V-inf, comme dans les exemples ci-dessous (voir aussi ex. 7) :

(18a) Des nouvelles un peu moins bonnes les firent précipiter leur départ (Gide, cité $\mathrm{BU})$

(18b) Les femmes les plus naïves ont un sens merveilleux qui (...) les fait ressaisir bientôt tout l'empire qu'elles ont laissé perdre (Martin du Gard, cité BU)

(18c) L'inquiétude naturelle aux malades qui les fait essayer sans cesse de nouveaux régimes (Bourget, cité BU)

Blanche-Benveniste (1978: 195) formule également l'hypothèse que l'alternance datif vs accusatif, là où elle se manifeste, est motivée par l'agentivité du sujet. Ainsi, les deux clitiques seraient possibles chaque fois qu'une double lecture est possible. Toutefois, l'analyse de Blanche-Benveniste semble se concentrer 
sur l'agentivité du sujet principal plutôt que celle du sujet de l'infinitif. Dans (19a-b) par exemple, l'opposition correspond au fait que dans (19a) Paul fait en sorte qu'elle pense à son chimpanzé (qu'elle ne doit pas oublier de nourrir), alors que dans (19b) Paul a un physique qui rappelle celui d'un chimpanzé. Le sujet Paul est donc + agentif dans le premier cas (il est la cause directe de V-inf) et -agentif (il est la cause indirecte de V-inf) dans le deuxième, ce qu'on peut schématiser comme suit :

\section{Lecture "A agentive" : "A faire X et X Cause B penser à son chimpanzé" \\ Lecture "A non agentive" : "A être X et X cause B penser à son chimpanzé"}

Dans le deuxième cas d'ailleurs, faire penser est quasiment lexicalisé, la phrase équivaut à 'il lui rappelle un chimpanzé' (par son physique) :

(19a) Paul la fait penser à son chimpanzé

(19b) Paul lui fait penser à son chimpanzé

Dans l'exemple suivant, l'accusatif est préférable parce que le sujet principal est hautement agentif alors que celui de V-inf ne l'est aucunement, il n'est que patient de faire et prend par conséquent l'accusatif; (20b) est douteux parce que le verbe statif ressembler est incompatible avec le "patient responsable » (Herslund 1997) qui correspond au datif : ${ }^{12}$

(20a) Je la fais ressembler à sa sœur (dans mon tableau)

(20b) ?? Je lui fais ressembler à sa sœur

Comme le suggèrent les exemples ci-dessus, un des facteurs qui rend l'analyse de la structure causative malaisée (et qui pourrait être à la base de la confusion chez le locuteur de l'accusatif et du datif) réside sans doute dans le fait qu'il y a toujours deux éléments potentiellement agentifs en jeu, et qu'ils peuvent l'être à des degrés divers selon le cas (cf. infra).

Avant de passer à l'examen des données concernant penser et réfléchir, encore une remarque importante. Tasmowski \& Van Oevelen (1987 : 56) notent de façon intéressante à propos des exemples (19a-b) qu'il « existe indubitablement des variantes idiolectales ici. Pour nous [= T \& VO], les gloses sont exactement inverses (nous soulignons) ». Selon les mêmes auteurs, de surcroît, « Certains jugent que (a) mais non (b) possède les deux lectures. Ils interprètent alors (b) avec la glose de (a) ». Autrement dit, tout semble possible et le moins qu'on puisse dire est que l'interprétation de ce genre de phrases ne fait pas l'unanimité des locuteurs, même linguistes. Autrement dit, la zone d'alternance entre datif / accusatif dans la structure causative en faire semble instable. C'est ce que suggèrent également les données que nous analysons ci-après.

\section{Analyse}

Les exemples sont tirés de Google, ils contiennent tous soit penser à soit réfléchir à : nous avons donc examiné deux structures syntaxiques identiques, transitives indirectes. Les deux verbes prennent un sujet + humain. Penser admet également un complément d'objet direct dans sa valence, à l'encontre de réfléchir.

Du point de vue quantitatif, penser semble bien plus courant après faire que réfléchir: une même consultation de Google ${ }^{13}$ fournit 1424 attestations de faire penser, contre 82 attestations de faire réfléchir. Quant à la distribution entre datif et accusatif, les deux verbes présentent des proportions inverses : alors que pour faire penser, $80 \%$ des exemples apparaissent avec le datif (lui/leur) contre $20 \%$ avec l'accusatif (le/la/les), $82 \%$ des phrases contenant faire réfléchir prennent l'accusatif et $18 \%$ seulement le datif. Voici quelques exemples :

(21a) Elle m'a dit que mon ami lui faisait penser à moi et que sa femme lui faisait penser à son père !

(21b) Les élèves revoient les éléments du poème de la création; ils choisissent et expliquent un élément qui leur fait penser à Dieu 
(21c) Puis il se met ensuite à embrasser la pâte à modeler et me parler de son odeur qui lui fait penser à celle d'un bébé.

(22a) Il y a des gens qui disent que je les fais penser à Michel Serres. Je souris et je pense que ça vient des cheveux!

(22b) De même que le crépuscule le fait penser à Clélia, il peut aussi symboliser le point de changement dans la vie de Fabrice.

(22c ) Le ruisseau d'or la fait penser à l'argent.

(23a) Lors de cet entretien, la directrice évalue le profil de l'étudiant, lui fait réfléchir aux défis à relever et propose les solutions de suivi

(23b) La Vertu de Prudence est une qualité qui dirige l'homme vers le Bien et lui fait réfléchir aux moyens d' y arriver

(23c) Aussi, il leur fait réfléchir à d'autres solutions pour envisager un développement durable, tout en satisfaisant à leurs besoins

(24a) Elle sensibilise les enfants et les fait réfléchir à leurs habitudes de consommation

(24b) Vu leur âge c'est uniquement la situation qui les fait réfléchir à leur passé

(24c) Cette visite les fait réfléchir à d'autres possibilités d'orientation que les lycées généraux

La prépondérance de datifs avec penser qui contraste avec l'accusatif dominant dans le cas de réfléchir est un argument en faveur de l'hypothèse syntaxique de Dannell (1979) puisque seul penser admet également un objet direct. On notera toutefois que, comme l'a remarqué Skårup (1990) en parlant d'un exemple de M. Herslund $(1976: 246){ }^{14}$, ces cas avec datif sont syntaxiquement étonnants dans la mesure où il y a deux compléments indirects alors qu'il s'agit d'un seul verbe, complexe il est vrai : en principe un verbe n'est jamais accompagné de deux objets indirects à la fois.

Comme l'illustrent les exemples ci-dessus, le caractère \pm animé du sujet principal ne semble pas jouer de rôle décisif dans la distribution du datif/accusatif, ni dans le cas de penser ni dans celui de réfléchir d'ailleurs : on observe le datif comme l'accusatif quand le sujet de faire est animé ou inanimé. Nos données remettent ainsi en question la théorie de certains (Verhagen \& Kemmer, 1997 par ex.) pour qui la causation directe serait plutôt associée à des SN de type + animé, la causation indirecte aux SN de type animé.

Même si tous les objets de faire sont nécessairement de type + humain puisque les deux infinitifs penser et réfléchir sélectionnent par définition un sujet humain, on constate cependant une différence importante entre les deux : avec réfléchir, le sujet est nécessairement agentif, il est activement impliqué par sa pensée tandis qu'avec penser, le degré d'agentivité varie selon le cas, comme l'avait bien remarqué BlancheBenveniste au sujet des exemples (19a-b). Dans la première série d'exemples ci-dessous, le sujet de l'infinitif est bien l'agent de la pensée, alors que dans la deuxième série, il n'en est que le siège : dans ce cas faire penser est proche du sens 'évoquer, rappeler' et la causation est indirecte, passant souvent par le biais d'une image. Dans ce cas, on assiste à une lexicalisation de faire penser, analogue à celle qu'on observe dans faire comprendre, faire voir, etc. Ce qui est pourtant crucial, c'est que la causation directe vs indirecte ne semble pas être un critère décisif pour le choix du clitique avec faire penser puisqu'on trouve aussi bien le datif que l'accusatif dans les deux cas, comme le montrent (25a-d) et (26a-d):

(25a) il se trouve que celle-ci a très peu d'amis et se retrouve donc souvent seule ce qui la fait penser à son passé (25b) Aussi quand le petit prince fait de la montagne, il fait une expérience qui le fait penser aux gens. Au sommet de la montagne il écoute l'écho et il trouve ...

(25c) car la confrontation d'un enfant à la mort lui fait penser à sa propre mort, sans doute pour la première fois

(25d) L'enseignante leur fait penser aux documentaires qu'ils ont vus dans leur passé.

Etaient-ils équitables ? Auraient-ils dû l'être? 
(26a) Parce que la lumière des éoliennes la fait penser à un incendie

(26b) Pour la petite histoire, mes frangines me disent que cette photo les fait penser à Wilma Petersen, ou Gil Grissom du feuilleton télé « Les Experts »

(26c) Les hommes sont si attirés par les seins des femmes car ça les fait retourner en enfance, ça leur fait penser au sein de leur mère

(26d) Depuis quelque temps il lui dit qu'elle lui fait penser à la Gestapo ! Trop c'est trop pour Marie

Dans certains cas, les phrases sont indéterminées entre une lecture agentive et non agentive du sujet de l'infinitif, comme dans les exemples suivants ; on trouve dans ces cas également les deux clitiques :

(27a) Après avoir fait le ménage dans sa vie, voilà qu'elle s'attaque à ce qui lui fait penser à son ex

(27b) la mer évoque l'éveil de la nature, dans un paysage maritime, l'éveil de l'espoir dans un pays étranger qui le fait penser à sa patrie

$\mathrm{Si}$ on considère les proportions indiquées ci-dessus opposant les deux verbes penser et réfléchir, ce qui semble faire pencher pour le choix de l'accusatif est davantage l'agentivité du contrôleur de l'infinitif que celui du verbe faire (le sujet principal) puisque l'accusatif est prépondérant là où l'agent est nécessairement et directement impliqué (le cas de réfléchir). Cela dit, les exemples montrent clairement, nous semble-t-il, qu'il s'agit là d'une tendance plus que d'une règle.

\section{Conclusions}

Il est évident que de plus amples recherches, tant du point de vue de la taille des corpus que du choix des infinitifs se combinant avec faire sont indispensables pour étayer, ou infirmer, les observations présentées ci-dessus. Dans l'ensemble, pour les cas observés ici, l'instabilité entre le datif et l'accusatif semble plus importante que ne le laissent entendre les grammaires.

$\mathrm{Si}$, comme nous l'avons vu, les propriétés syntaxiques de l'infinitif qui suit faire jouent certainement un rôle dans l'alternance des clitiques, la complexité sémantique de la structure causative pourrait y être également pour beaucoup. En effet, comme toute causation met en cause au moins deux acteurs, chacun avec une agentivité relative, on conçoit que le continuum causatif comprenne une série de cas de figure se situant dans une zone intermédiaire où l'opposition entre accusatif et datif se neutralise.

Du point de vue référentiel, on peut en effet analyser une situation dans laquelle A fait que B mange sa soupe de plusieurs manières :

\begin{tabular}{|l|l|l|l|l|}
\hline & & A & B \\
\hline & & Agentif & Agentif & Volitionnel \\
\hline 1 & Je le (lui) fais manger sa soupe & + & - & - \\
\hline 2 & Je le (lui) fais manger sa soupe & + & - & + \\
\hline 3 & Je lui (le) fais manger sa soupe & + & + & - \\
\hline 4 & Je lui fais manger sa soupe & + & + & + \\
\hline
\end{tabular}

1 : B n'est pas en mesure physiquement de manger sa soupe et il n'en a pas la volonté (les grands malades, les bébés)

2 : B voudrait manger mais ne peut pas manger sa soupe sans une aide

3 : B peut la manger mais ne le veut pas

4 : je fais simplement penser à B qu'il devrait manger sa soupe parce qu'il a faim (les enfants qui peuvent manger seuls, qui ont faim, mais qui ne pensent pas à manger) 
Le datif est possible dans tous les cas mais on l'attendrait plus à partir 3 et même de 2, et il serait quasiment obligatoire dans 4 .

On comprend aisément qu'indépendamment des considérations syntaxiques, l'accusatif sera d'autant plus facilement «confondu » avec le datif que l'agent de V-inf porte le trait + humain, comme c'est le cas avec les verbes penser et réfléchir, le référent prototypique des datifs étant humain alors que le référent prototypique de l'accusatif porte le trait [-hum].

La confusion entre les deux cas observée ici n'est après tout pas si étonnante du point de vue typologique. Dans une langue proche du français, l'espagnol, on observe une neutralisation généralisée des clitiques datif vs accusatif, indépendamment de la structure causative : en effet on y utilise le datif pour l'objet « direct » appelé « accusatif prépositionnel » quand il porte le trait [+hum] et inversement, et dans une moindre mesure, l'accusatif pour l'objet dont la fonction est celle d' "objet indirect", comme dans:

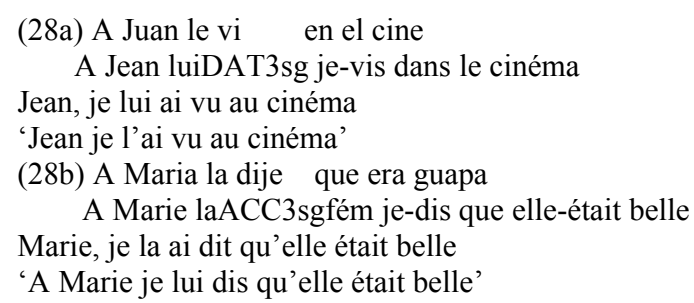

\section{Références}

Blanche-Benveniste, C., Deulofeu, J., Stefanini, J. et Van Den Eynde, K. (1987). Pronom et syntaxe: l'approche pronominale et son application au français. Paris. SELAF.

Blanche-Benveniste, C. (2007). Les énoncés à causatifs réfléchis. In: Rousseau, A., Bottinau, D. et Roulland, D. (éds). L'énoncé réfléchi. Rennes: Presses Universitaires de Rennes, 155-173.

Comrie, B. et Polinsky, C. éds (1993). Causatives and Transitivity. Amsterdam: J. Benjamins.

Dannell, K.J. (1979). Remarques sur la construction dite causative. Faire (laisser, voir, entendre, sentir) + Infinitif. Stockholm: Almqvist \& Wiksell.

Dixon, R.M.W. (2000). A typology of causatives: form, syntax and meaning. In Dixon, R.M.W et Aikhenvald, A. (éds) Changing valency. Cambridge: CUP, 30-82.

Fournier, N. (1998). Grammaire du français classique, Paris, Belin.

Grevisse-Goosse, A. (2008). Le Bon Usage. Bruxelles: De Boeck-Duculot.

Herslund, M. (1988). Le datif en français. Louvain: Peeters.

Herslund, M. (1990). Remarques sur la construction causative: Réponse à P. Skårup. Aarhus Universitet: Romansk Institut, (Pré)publications.

Hulk A. (1984). De la syntaxe du pronom en dans la construction quantitative. Revue Québecoise de linguistique 3. 167-199.

Kayne, R. (1977). Syntaxe du français. Paris: Seuil.

Lamiroy, B. (2000). De la syntaxe comparée des langues romanes: du passif au datif possessif. In: Schösler, L. (éd.) Le passif. Etudes romanes, $\mathrm{n}^{\circ}$ spécial 45, 135-154.

Lazard, G. (1994). L'actance. Paris: PUF.

Le Goffic, P. (1993). Grammaire de la phrase française. Paris : Hachette.

Perlmutter, D. (1983). Studies in Relational Grammar, vol. I. Chicago: Chicago University Press.

Riegel, M., Pellat, J.-C. et Rioul, R. (1996). Grammaire méthodique du français. Paris : PUF. 
Rouveret, A. et Vergnaud, J.R. (1980). Specifying Reference to the Subject: French Causatives and Conditons on Representations. Linguistic Inquiry, 11, 97-202.

Shibatani, M. (1976). The grammar of causative constructions. In: Shibatani, M. (ed.) Syntax and Semantics 6. New York: Academic Press, 1-42.

Shibatani, M. (2002). The causative continuum. In: Shibatani, M. (ed.) The Grammar of Causation and Interpersonal Manipulation. Amsterdam: J. Benjamins, 136-177.

Skårup, P. (1985). Compte-rendu de Dannell 1979.

Skårup, P. (1990). Faire + Infinitif selon Michael Herslund. Aarhus Universitet: Romansk Institut, (Pré)publications.

Tasmowski-De Ryck L. (1984). ?*Lui faire téléphoner quelqu'un d'autre: une stratégie?, Lingvisticae Investigationes VIII : 2, 403-427.

Tasmowski-De Ryck L. (1985). Faire infinitif. In : L. Melis et al. (eds), Les constructions de la phrase française. Gent: Communication et Cognition, 233-365.

Tasmowski-De Ryck L. et Van Oevelen, H. (1987). Le causatif pronominal. Revue Romane, 22, 40-58.

Tayalati, F. (2008). La distinction ergatif/inergatif et son incidence sur le placement des clitiques datifs dans les constructions causatives avec faire et rendre. Probus, 20-2, 301-321.

Van Belle, W. and W. Van Langendonck éds. (1996-1997). The Dative, 2 Volumes. Amsterdam/Philadelphia: John Benjamins.

Verhagen, A. \& Kemmer, S. (1997). Interaction and causation: Causative constructions in modern standard Dutch, Journal of Pragmatics, 27, 1, 61-82.

${ }^{1}$ La littérature consacrée à cette construction étant très vaste, on comprendra que dans les limites de cet article, seules les sources pertinentes seront mentionnées.

${ }^{2}$ Nous utilisons, à la suite de Grevisse-Goosse (2008) et de Blanche-Benveniste (2007) les termes d'accusatif et de datif pour des raisons de commodité.

${ }^{3}$ La structure causative existant dans un très grand nombre de langues (Shibatani 1976, 2002), de nombreux linguistes se sont efforcés d'en fournir une définition universelle (par ex. Talmy 1976, Wierzbicka 1998, Wolff 2008).

${ }^{4}$ Dans beaucoup de langues, comme en français, la structure causative partage cette propriété avec les verbes de perception.

${ }^{5}$ La structure Paul l'a fait y répondre n'est pas impossible mais, comme le remarque Blanche-Benveniste (2007), faire est peu attesté dans ce type de construction; il l'est en revanche bien plus dans la construction où il fait partie d'un complexe verbal et où il a le statut d'auxiliaire causatif ou opérateur diathétique (Riegel 1996 :229).

${ }^{6}$ Selon Perlmutter (1988 : 379), cette règle «d'avancement» caractéristique des structures à clause union (dont la causative) s'observe dans nombre de langues. Comrie et Polinsky (1993) partagent cet avis.

${ }^{7}$ Tout ce qui appartient au langage d'Internet ne reflète pas automatiquement le code oral. Toutefois, la langue des "chats" et des "blogs", où les utilisateurs discutent sous forme de dialogue, reproduit un type de langage proche de l'oral. L'utilisation de ce genre de sources rappelle la pratique de grammairiens traditionnels (tels Grevisse-Goosse 2008) qui citent des passages de pièces de théâtre pour illustrer certains usages de la langue parlée.

${ }^{8}$ Ainsi, pour je le/la/les fais manger un/une ... nous avons obtenu un seul cas, alors que je lui/leur fais manger un/une ... donne 41 occurrences. Avec le verbe construire, les proportions sont de 2 accusatifs vs 14 datifs. Il est évident que les chiffres varient en fonction de l'infinitif qui suit : nos données ne sont que le résultat de sondages ponctuels et demanderaient évidemment à être complétées par une recherche systématique sur un corpus de grande envergure.

${ }^{9}$ Dans cet exemple, on a bien affaire au sens agentif de sentir 'renifler' et non pas au sens ergatif où le sujet a le rôle de thème, comme ce serait le cas dans Cette veste sent le tabac.

${ }^{10}$ Les cas où le référent de l'objet de faire est inanimé, comme dans l'exemple de Kayne (1977:240), sont rares pour des raisons évidentes dues au sémantisme de la construction causale : l'objet de la causation est par définition un 
patient « responsable » (Herslund 1988 ; voir aussi Tasmowski et Van Oevelen 1987), c'est-à-dire qu'il doit être doué d'une autonomie relative pour exécuter l'action de V-inf.

${ }^{11}$ En fait, Damourette \& Pichon (1936 § 2047) proposent, en d'autres termes, une analyse tout à fait analogue à celle de Herslund : d'après eux, dans Le chirurgien fit opérer ce malade à son interne « la fin principale (...) c'est de faire que l'interne opère », tandis que dans Le chirurgien fit opérer ce malade par son interne «la fin principale (...) c'est que le malade soit opéré et par là débarrassé de son mal». Kayne (1977: 227ss) et Tasmowski \& Van Oevelen (1987) proposent une analyse analogue .

${ }^{12}$ Nous ne partageons pas le jugement de Blanche-Benveniste (2007), pour qui le verbe ressembler serait simplement incompatible avec la construction causative.

${ }^{13}$ Il s'agit d'une consultation faite le 11/11/2009 à partir d'une requête sur le/la/les fait penser/réfléchir à/aux .

${ }^{14}$ L'exemple dont il s'agit est "la luminosité des feux arrière lui fit penser à une voiture allemande". 